\begin{tabular}{|c|l|}
\hline Title & Finite dimensional solution sets of extremal problems in $\mathrm{H} 1$ \\
\hline Author(s) & Inoue, Junji; Nakazi, Takahiko \\
\hline Citation & Hokkaido University Preprint Series in Mathematics, 171, 1-10 \\
\hline Issue Date & 1992-11 \\
\hline DOI & 10.14943/83315 \\
\hline Doc URL & http://hdl.handle.net/2115/68917 \\
\hline Type & bulletin (article) \\
\hline File Information & pre171.pdf \\
\hline
\end{tabular}

Instructions for use 
Finite Dimensional Solution Sets of Extremal Problems in $\mathrm{H}^{1}$

J. Inoue and T. Nakazi

Series $\sharp 171$. November 1992 


\section{HOKKAIDO UNIVERSITY \\ PREPRINT SERIES IN MATHEMATICS}

$\sharp 144$ : K. Kubota, K. Mochizuki, On small data scattering for 2-dimensional semilinear wave equations, 22 pages. 1992.

\$145: T. Nakazi, K. Takahashi, Hyponormal Toeplitz operators and extremal problems of Hardy spaces, 30 pages. 1992.

\#146: N. Hayashi, T. Ozawa, Remarks on nonlinear Schrödinger equations in one space dimension, 10 pages. 1992.

$\sharp 147$ : M. Sato, Interface evolution with Neumann boundary condition, 16 pages. 1992.

\#148: Y. Okabe, Langevin equations and causal analysis, 49 pages. 1992.

\#149: Y. Giga, S. Takahashi, On global weak solutions of the nonstationary two-phase Stokes flow, 25 pages. 1992.

$\sharp 150: \quad$ G. Ishikawa, Determinacy of envelope of the osculating hyperplanes to a curve, 9 pages. 1992.

$\sharp 151: \quad$ G. Ishikawa, Developable of a curve and determinacy relative to osculation-type, 15 pages. 1992.

$\sharp 152$ : H. Kubo, Global existence of solutions of semilinear wave equations with data of non compact support in odd space dimensions, 25 pages. 1992.

$\sharp 153$ : Y. Watatani, Lattices of intermediate subfactors, 33 pages. 1992.

$\sharp 154$ : T. Ozawa, On critical cases of Sobolev inequalities, 11 pages. 1992.

$\sharp 155$ : M. Ohnuma, M. Sato, Singular degenerate parabolic equations with applications to geometric evolutions, 20 pages. 1992

$\sharp 156$ : S. Izumiya, Perestroikas of optical wave fronts and graphlike Legendrian unfoldings, 13 pages. 1992.

$\sharp 157$ : A. Arai, Momentum operators with gauge potentials, local quantization of magnetic flux, and representation of canonical commutation relations, 11 pages. 1992.

$\sharp 158$ : S. Izumiya, W.L. Marar, The Euler number of a topologically stable singular surface in a 3-manifold, 11 pages. 1992.

\# 159: T. Hibi, Cohen-Macaulay types of Cohen-Macaulay complexes, 26 pages. 1992.

$\sharp 160$ : A. Arai, Properties of the Dirac-Weyl operator with a strongly singular gauge potential, 26 pages. 1992.

\#161: A. Arai, Dirac operators in Boson-Fermion Fock spaces and supersymmetric quantum field theory, 30 pages. 1992.

$\sharp 162$ : S. Albeverio, K. Iwata, T. Kolsrud, Random parallel transport on surfaces of finite type, and relations to homotopy, 8 pages. 1992.

$\sharp 163$ : S. Albeverio, K. Iwata, T. Kolsrud, Moments of random fields over a family of elliptic curves, and modular forms, 9 pages. 1992.

\# 164: Y. Giga, M. Sato, Neuman n problem for singular degenerate parabolic equations, 12 pages. 1992.

\# 165: J. Wierzbicki, Y. Watatani, Commuting squares and relative entropy for two subfactors, 18 pages. 1992.

$\sharp 166$ : Y. Okabe, A new algorithm driven from the view-point of the fluctuation-dissipation theorem in the theory of $\mathrm{KM}_{2} \mathrm{O}$-Langevin equations, 13 pages. 1992.

\$167: Y. Okabe, H. Mano and Y. Itoh, Random collision model for interacting populations of two species and its strong law of large numbers, 14 pages. 1992.

$\sharp 168$ : A. Inoue, On the equations of stationary precesses with divergent diffusion coefficients, 25 pages. 1992.

$\sharp 169: \quad$ T. Ozawa, Remarks on quadratic nonlinear Schrödinger equations, 19 pages. 1992.

$\sharp 170: \quad$ T. Fukui, Y. Giga, Motion of a graph by nonsmooth weighted curvature, 11 pages. 1992. 


\title{
Finite Dimensional Solution Sets of Extremal Problems in $H^{1}$
}

\author{
Jyunji Inoue* and Takahiko Nakazi *
}

\section{Dedicated to Professor Tuyoshi Ando on his 60th birthday}

For a non-zero function $f$ in $H^{1}$, the classical Hardy space on the unit circle, put

$$
S_{|f| / f}=\left\{g \in H^{1}:\|g\|_{1}=1, \arg f\left(e^{i t}\right)=\arg g\left(e^{i t}\right) \text { a.e.t }\right\},
$$

then $S_{|f| / f}$ is the set of extremal functions of a well known linear extremal problem in $H^{1}$. It is known and easy to see that if $f^{-1}$ belongs to $H^{1}$ then the dimension of $\left\langle S_{|f| / f}\right\rangle$, the linear span of $S_{|f| / f}$, is one. A simple example shows that even if $f^{-1}$ belongs to $H^{p}$ for some $p(0<p<1)$, the dimension of $\left\langle S_{|f| / f}\right\rangle$ may be infinite. On the other hand, a sophisticated example (will be shown in this paper) shows that even if $f^{-1}$ locally belongs to $H^{1}$ on the unit circle except a finite set, the dimension of $\left\langle S_{|f| / f}\right\rangle$ may be infinite. In this paper it is shown that if $f \in H^{1}$ has the properties such that $f^{-1}$ locally belongs to $H^{1}$ on the unit circle except a finite set and that $f^{-1} \in H^{p}$ for some $p>0$, then the dimension of $\left\langle S_{|f| / f}\right\rangle$ is finite.

\section{Introduction}

Let $U$ be the open unit disc with the boundary $T=\partial U$. An analytic function $f$ on $U$ is said to belong to $N$ if $\sup _{0 \leq r<1} \int_{-\pi}^{\pi} \log ^{+}\left|f\left(r e^{i t}\right)\right| d t<\infty$.

\footnotetext{
*This research was partially supported by Grant-in-Aid for Scientific Research, Ministry of Education.
} 
Each function $f$ in $N$ has a boundary value $f\left(e^{i t}\right)=\lim _{r \rightarrow 1-} f\left(r e^{i t}\right)$ a.e.t. The set of all $f$ in $N$ satisfying

$$
\lim _{r \rightarrow 1-} \int_{-\pi}^{\pi} \log ^{+}\left|f\left(r e^{i t}\right)\right| d t=\int_{-\pi}^{\pi} \log ^{+}\left|f\left(e^{i t}\right)\right| d t
$$

will be denoted by $N^{+}$. The Hardy space $H^{p}(U)(0<p \leq \infty)$ is the subspace of all $f$ in $N^{+}$whose boundary function $f\left(e^{i t}\right)$ belongs to $L^{p}(T)$, the usual Lebesgue space on $T$. Let $1 \leq p, q \leq \infty, 1 / p+1 / q=1$ and $\varphi \in L^{q}$. The bounded linear functional $T_{\varphi}$ on $H^{p}$ with kernel function $\varphi$ is defined by

$$
T_{\varphi}(f)=\int_{-\pi}^{\pi} f\left(e^{i t}\right) \varphi\left(e^{i t}\right) d t / 2 \pi \quad\left(f \in H^{p}\right),
$$

and the norm of $T_{\varphi}$ is given by $\left\|T_{\varphi}\right\|=\sup \left\{\left|T_{\varphi}(f)\right|: f \in H^{p},\|f\|_{p} \leq 1\right\}$. When $T_{\varphi}$ is nonzero, we put $S_{\varphi}=\left\{f \in H^{p}: T_{\varphi}(f)=\left\|T_{\varphi}\right\|,\|f\|_{p} \leq 1\right\}$. The set $S_{\varphi}$ is the intersection of the hyperplane $\left\{f: T_{\varphi}(f)=\left\|T_{\varphi}\right\|\right\}$ with the unit ball of $H^{p}$. When $1<p \leq \infty$, the structure of the set $S_{\varphi}$ is simple since the set $S_{\varphi}$ consists of exactly one point. But when $p=1$ the situation is quite different from the former case.

In 1958, deLeeuw and Rudin [1] studied the structure of $S_{\varphi}$, and among many fundamental results concerning extremal problems, they completely described the set $S_{\varphi}$ when the function $\varphi$ can be extended analytically to the set $\{|z| \geq r\}$ for some $r<1$.

Since then, the study of $S_{\varphi}$ has been continued by several authors. Nakazi, the one of the authors of this paper, showed in [7] that if $S_{\varphi}$ is a weak*compact subset of $H^{1},<S_{\varphi}>$ has finite dimension, and that the set $S_{\varphi}$ can be described completely in the same manner as deLeeuw and Rudin carried out in [1]. For continuous kernel $\varphi$, the set $S_{\varphi}$ is weak*-compact, and hence Nakazi's result above can be applied. It is easy to see that the above deLeeuw-Rudin's case is contained in this case.

Let $\varphi=|f| / f$ for some nonzero function $f \in H^{1}$. Even if $f$ is a polynomial, $\varphi$ may not be continuous but $\left\langle S_{\varphi}\right\rangle$ is finite dimensional. We are interested in the problem to decide the functions $f \in H^{1}$ such that $<S_{\varphi}>$ for $\varphi=|f| / f$ has finite dimension. If $f^{-1}$ belongs to $H^{1}$ then $S_{|f| / f}=\{f\}$, that is the dimension of $\left\langle S_{|f| / f}\right\rangle$ is one ([7]). We want to relax the hypothesis on $f^{-1}$. It is known that $f^{-1}$ belongs to $N_{+}$if and only if $f$ is outer. Hence without loss of generality we may assume that $f^{-1}$ belongs 
to $N_{+}$. Regarding $N_{+} \cap L^{p}=H^{p}$, we want to define a local version of the Hardy space $H^{p}$.

For each $c \in T, I_{c}$ denotes an arc in $T$ which contains $c$ at the center of $I_{c}$ and put $L^{p}\left(I_{c}\right)=\left\{f: f\right.$ is measurable on $T$ and $\left.\int_{I_{c}}\left|f\left(e^{i t}\right)\right|^{p} d t<\infty\right\}$.

Definition. For the function $g \in N_{+}$we say that $g$ locally belongs to $H^{p}$ at $c \in T$ when $g \in N_{+} \cap L^{p}\left(I_{c}\right)$ for some $I_{c}$.

If $g$ locally belongs to $H^{p}$ at every point on $T$, then it is easy to see that $g$ belongs to $H^{p}$. We can conjecture that if $f \in H^{1}$ and $f^{-1} \in N_{+}$locally belongs to $H^{1}$ on the unit circle except a finite set, then the linear span of $S_{|f| / f}$ is finite dimensional. However, we have a counter example which will be given in $\S 3$. In the above conjecture, if $f^{-1} \in H^{p}$ for some $0<p<1$ instead of $f^{-1} \in N_{+}$then the conjecture is valid. This is our main result which will be proved in $\S 2$.

In the proof of the main result (Theorem 1) the following Lemma 1 , which may be some independent interest, plays an essential role. If $A=\emptyset$ in (b) of Lemma 1 , it follows easily that $F$ must be constant. This is the Neuwirth-Newman Theorem [8].

Lemma 1. Let $F$ be a function in $N^{+}$such that

(a) $F$ belongs to $H^{p}$ for some $p>0$,

(b) $F$ locally belongs to $H^{1 / 2}$ except a finite set $A$ of $T$,

(c) $F$ is outer and $F\left(e^{i t}\right) \geq 0$ a.e. on $T$.

Then $F$ can be extended to a rational function.

\section{The main theorem and its corollaries}

In this section the main theorem in this paper and its corollaries will be proved. The proof of Lemma 1 which plays an essential role in the proof will be given in $\$ 4$. 
Notation : In the rest of this paper, the notation [a] for a real number $a$ is used to express the largest integer which is equal or less than $a$.

Theorem 1. Suppose $f$ is a nonzero function in $H^{1}$. If $f^{-1}$ locally belongs to $H^{1}$ on the unit circle except a finite set $A$ of $T$ and $f^{-1} \in H^{p}$ for some $p>0$, then the linear span of $S_{|f| / f}$ has finite dimension.

Moreover, if $A=\left\{\alpha_{j}: j=1,2, \ldots, n\right\}$ and if $p_{j}=\sup \left\{q: f^{-1}\right.$ locally belongs to $H^{q}$ at $\left.\alpha_{j}\right\}$ for $j=1,2, \ldots, n$, then we have

$$
\operatorname{dim}<S_{|f| / f}>\leq 1+\sum_{j=1}^{n} 2\left[\frac{p_{j}+1}{2 p_{j}}\right]
$$

Proof. If $S_{|f| / f}=\{f /\|f\|\}$, the theorem is trivial, and we suppose that $S_{|f| / f} \neq\{f /\|f\|\}$. Let $g$ be an arbitrary outer function in $S_{|f| / f}$ and put $F(z)=g(z) / f(z) \quad(z \in U)$. We claim that $F(z)$ has the following properties:

(a) $F$ locally belongs to $H^{1 / 2}$ except a finite set $A$ of $T$,

(b) At $z=\alpha_{j} \in A, F$ locally belongs to $H^{q}$ for each $q\left(0<q<\frac{p_{j}}{1+p_{j}}\right)$,

(c) $F$ is outer and $F\left(e^{i t}\right) \geq 0$ a.e. on $T$.

(a) and (b) are deduced from the conditions on $f$ and $g$ by using the Hölder's inequality, and (c) is obvious from the conditions on $f$ and $g$.

By (a), (b) and (c) using Lemma 1 of $\S 4, F$ can be extended to a rational function with poles only at points in $A$. We also express this extension by the same symbol $F(z)$. Note that if $\alpha_{j}$ is a pole of order $n_{j}$ of $F, n_{j}$ must be an even integer by $(c)$, and hence we have $n_{j}=2 m_{j}$ with $m_{j} \leq\left[\frac{p_{j}+1}{2 p_{j}}\right]$. Since $F(z)$ has no zeros on $U$, its zeros are all on $T$. Therefore, with some $\gamma>0$, we can represent $F(z)=g(z) / f(z)$ in the form

$$
F(z)=\frac{\gamma \prod_{k=1}^{N}\left(z-z_{k}\right)\left(\overline{z_{k}} z-1\right)}{\prod_{j=1}^{n}\left(z-\alpha_{j}\right)^{m_{j}}\left(1-\overline{\alpha_{j}} z\right)^{m_{j}}}
$$

where $N=m_{1}+\ldots+m_{n}$ and $z_{k} \in T \quad j=1, \ldots, N$. From (1), it follows that each outer function $g$ in $S_{|f| / f}$ has the expression of the form

$$
g(z)=\gamma f(z) \frac{\prod_{k=1}^{N}\left(z-z_{k}\right)\left(\overline{z_{k}} z-1\right)}{\prod_{j=1}^{n}\left(z-\alpha_{j}\right)^{m_{j}}\left(1-\overline{\alpha_{j}} z\right)^{m_{j}}} \quad(z \in U),
$$

with the relations $m_{j} \leq\left[\frac{p_{j}+1}{2 p_{j}}\right] j=1,2, \ldots, n$. 
By using the Nakazi's results in [7], we can conclude that

$$
\operatorname{dim}<S_{|f| / f}>\leq 1+\sum_{j}^{n} 2\left[\frac{p_{j}+1}{2 p_{j}}\right]
$$

and this completes the proof.

In the following three corollaries of Theorem 1, we need not assume $f^{-1} \in$ $H^{p}$ for some $p>0$.

Corollary 1. Suppose $f$ is a schlicht function in $H^{1}$. If $f^{-1}$ locally belongs to $H^{1}$ on $T$ except a finite set $A$, then $\operatorname{dim}<S_{|f| / f}>\leq \max \{3,2|A|+1\}$, where $|A|$ denotes the number of elements of $A$.

Proof. We divide the situation into two cases: (i) $f(z) \neq 0$ for each $z \in U$, (ii) There exists $\alpha \in U$ such that $f(\alpha)=0$. In the case (i), $f^{-1}$ is a schlicht function and hence belongs to $H^{p}$ for all $p(0<p<1 / 2)$ at each point of $A$. Thus we have $\operatorname{dim}\left\langle S_{|f| / f}>\leq 2|A|+1\right.$ by Theorem 1. In the case (ii), we can represent $f$ in the form $f(z)=(z-\alpha) g(z)$ with $g^{-1}$ is a bounded analytic function on $U$, and hence $g /\|g\|_{1}$ is an exposed point of the unit ball of $H^{1}$. Hence we get $\operatorname{dim}\left\langle S_{|f| / f}\right\rangle=3$ (cf. [7]). Thus the proof is complete.

Corollary 2. Suppose $f$ is a nonzero function in $H^{1}$. If there exists a nonzero function $h \in H^{1}$ such that $h f$ is an analytic polynomial of degree $n$, then $\operatorname{dim}\left\langle S_{|f| / f}\right\rangle \leq 2 n+1$.

Proof. Let $f=q g$ be a factorization with an inner function $q$ and an outer function $g$, and put $s=h f$. Since $s$ is an analytic polynomial of degree $n$, we can represent $s$ in the form $s(z)=\beta \prod_{j=1}^{L}\left(z-\alpha_{j}\right)^{m_{j}}$, where $\alpha_{1}, \ldots, \alpha_{L}, \beta \in C$, and $m_{1}+\ldots+m_{L}=n$. It is easy to see that $q$ is a finite Blaschke product whose zeros are contained in $A \cap U$, where $A=\left\{\alpha_{1}, \ldots, \alpha_{L}\right\}$, and $\alpha_{j}$ may be a zero of $q$ with order at most $m_{j}$. On the other hand, $g^{-1}(=q h / s)$ locally belongs to $H^{1}$ at each point of $T \backslash A$, and locally belongs to $H^{q}$ with $0<q<m_{j}^{-1} /\left(m_{j}^{-1}+1\right)$ at each point of $\alpha_{j} \in T \cap A$. Hence we can apply Theorem1 to $g$, and get $\operatorname{dim}<S_{|g| / g}>\leq 1+\sum_{\left|\alpha_{j}\right|=1} 2\left[\frac{m_{j}+2}{2}\right]$. Thus we have

$$
\operatorname{dim}<S_{|f| / f}>\leq \sum_{\alpha_{j} \in U} 2 m_{j}+1+\sum_{\left|\alpha_{j}\right|=1} 2\left[\frac{m_{j}+2}{2}\right] \leq 1+2 n .
$$

This completes the proof. 
Corollary 3. Suppose $f \in H^{1}$ is continuous and $\Re[f(z)] \geq 0$ on $\bar{U}$. If $f$ has at most a finite number of zeros on $T$, the dimension of $\left\langle S_{\left|f^{n}\right| / f^{n}}\right\rangle$ is finite for any positive integer $n$.

Proof. Since $\Re[f(z)] \geq 0$ on $U$, we have $\Re\left[f^{-1}(z)\right] \geq 0$ on $U$, and hence $f^{-1}$ belongs to $H^{p}$ for each $p<1$. Therefore, $f^{-n}$ belongs to $H^{p}$ for some $p>0$, and $f^{-n}$ locally belongs to $H^{1}$ except a finite number of points on $T$. Thus the result follows from Theorem 1 .

If $q$ is an inner function and $f=1-q$ then $\Re f$ is nonnegative a.e. on $T$ and hence $f /\|f\|_{1}$ is an exposed point of the unit ball of $H^{1}$, that is $\operatorname{dim}\left\langle S_{|f| / f}\right\rangle=1$. If $q$ is a finite Blaschke product then by Corollary 3 $\left.\operatorname{dim}<S_{\left|f^{2}\right| / f^{2}}\right\rangle<\infty$. However, it is easy to see that if $q$ is not a finite Blaschke product then $\operatorname{dim} S_{\left|f^{2}\right| / f^{2}}=\infty$.

If $g \in H^{1}$ is continuous and $|g(z)| \leq 1$ on $\bar{U}$, and $f=1-g$, then $\Re f$ is nonnegative. If the peak set of $g$ is a finite set on the unit circle, then by Corollary $3 \operatorname{dim} S_{\left|f^{n}\right| / f^{n}}<\infty$ for any positive integer $n>0$. A well known Rudin-Carleson theorem [3] tells us how to construct such a $g$.

Corollary 4. If $q(z)$ is an inner function such that i) $q(z)$ is not a finite Blaschke product ii) The singular support of $q(z)$ is a finite set $A$. Then for each number $\alpha \in T, B=\{z \in T \backslash A: q(z)=\alpha\}$ is an infinite set.

Proof. If $B$ were a finite set, $f=(\alpha-q)^{2}$ would satisfy the conditions of Theorem 1 and hence $\operatorname{dim}\left\langle S_{|f| / f}\right\rangle$ must be finite. But it is well known and easy to prove that if $b(z)$ is an inner function which is not a finite Blaschke product, we have $\operatorname{dim}\left\langle S_{\left|(\alpha-b)^{2}\right| /(\alpha-b)^{2}}\right\rangle=\infty$. Thus we get a contradiction, which completes the proof.

\section{Examples}

In this section we give counter examples which we mentioned in the abstract and $\S 1$. That is, to get the conclusion we cannot omit in Theorem 1 either of (a) and (b) below:

(a) $f(z)$ locally belongs to $H^{1}$ except finite points of $T$,

(b) $f^{-1} \in H^{p}$ for some $p>0$. 
Example 1. Let $0<p<1$ and let $b$ be an infinite Blaschke product on the unit disc such that its zeros converge to 1 . Choose $p^{\prime}$ such that $0<p<p^{\prime}<1$ and put $f=(1-b)^{1 / p^{\prime}}$. We claim that $f$ satisfies (b) of the conditions above, but $\operatorname{dim}\left\langle S_{\left|(1-b)^{2}\right| /(1-b)^{2}}\right\rangle=\infty$.

Indeed, since $\left(f^{-1}\right)^{p}=(1-b)^{-p / p^{\prime}}$ with $p / p^{\prime}<1$, we have $f^{-1} \in H^{p}$, and since $f=(1-b)^{2}(1-b)^{-2+\left(1 / p^{\prime}\right)}$ with $(1-b)^{-2+\left(1 / p^{\prime}\right)} \in H^{1}$ we have $\operatorname{dim}\left\langle S_{|f| / f}\right\rangle=\infty$. Note that $f$ doesn't satisfy (a) of the conditions above by Corollary 4 .

Example 2. (cf. [5]) Let $f(z)$ be a function defined by

$$
F(z)=\prod_{k=1}^{\infty} \frac{\left(z-\alpha_{k}\right)\left(1-\bar{\alpha}_{k} z\right)}{(z-1)(1-z)} \quad(z \in U)
$$

where $\alpha_{k}=e^{-i / k^{2}}, k=1,2,3, \ldots$ It is easy to see that the right hand side infinite product in (2) converges uniformly on each compact set of $C \backslash\{1\}$, and $F\left(e^{i t}\right) \geq 0$ on $T \backslash\{1\}$. Moreover $F(z)$ is an outer function. To see this, we consider $\log F(z)$ on $\bar{U} \backslash\{1\}$ such that $\Im[\log F(-1)]=0$. Since $\Im\left[\log F\left(e^{i t}\right)\right]$ is a monotone decreasing step function on $(0,2 \pi)$, with jumps $2 \pi$ at $t=1 / k^{2}(k=1,2, \ldots)$, and hence $\Im[\log F]$ is a real harmonic function belonging to the Zygmund's class. Therefore the harmonic conjugates of $\Im[\log F]$ are contained in $h^{1}$ (cf. [6]), which implies that $F(z)$ is outer.

Choose $\varepsilon_{k}>0$ so that

$$
\begin{aligned}
& \frac{1}{k^{2}}>\frac{1}{k^{2}}-\varepsilon_{k}>\frac{1}{(k+1)^{2}}+\varepsilon_{k+1}, \\
& \left|F\left(e^{i t}\right)\right| \leq 1 \quad t \in\left(\frac{1}{k^{2}}-\varepsilon_{k}, \frac{1}{k^{2}}+\varepsilon_{k}\right) \quad k=1,2, \ldots
\end{aligned}
$$

and put $\Omega=\bigcup_{k=1}^{\infty}\left(\frac{1}{k^{2}}-\varepsilon_{k}, \frac{1}{k^{2}}+\varepsilon_{k}\right)$. If we define a function $g$ on $T \backslash\{1\}$ by

$$
g\left(e^{i t}\right)= \begin{cases}\min \left\{\frac{1}{F\left(e^{i t}\right),}, 1\right\}: & t \in[0,2 \pi) \backslash \Omega \\ \frac{1}{\varepsilon_{k} k^{4}}: & t \in\left(\frac{1}{k^{2}}-\varepsilon_{k}, \frac{1}{k^{2}}+\varepsilon_{k}\right), \quad k=1,2, \ldots\end{cases}
$$

then $\log g\left(e^{i t}\right)$ belongs to $L^{1}(T)$. Using this $g\left(e^{i t}\right)$ we define an outer function $f(z) \in H^{1}$ by

$$
f(z)=\exp \int_{0}^{2 \pi} \frac{e^{i t}+z}{e^{i t}-z} \log g\left(e^{i t}\right) d t / 2 \pi . \quad(z \in U)
$$


Then it is easy to see that $f(z)$ locally belongs to $H^{1}$ except one point $z=1$. But since $f(z) F(z) \in H^{1}$ and $\arg f\left(e^{i t}\right)=\arg f\left(e^{i t}\right) F\left(e^{i t}\right)$ a.e. on $[0,2 \pi)$ we can see that

$$
\operatorname{dim}<S_{|f| / f}>=\operatorname{dim}<S_{|f F| / f F}>=\infty .
$$

\section{Proof of Lemma 1}

In this section, we prove Lemma 1 used in the proof of the main theorem in $\S 2$. In the following, $C^{+}$and $C_{+}$denote the half planes given by $C^{+}=$ $\{z: \Im z>0\}$ and $C_{+}=\{z: \Re z>0\} . H^{p}\left(C^{+}\right)$(resp. $H^{p}\left(C_{+}\right)$) is the Hardy space on $C^{+}$(resp. $C_{+}$) in the usual sense (cf. [3, p.51]). $\psi(z)$ denotes the linear fractional transformation defined by $\psi(z)=(z-i) /(z+i)$, which maps $C^{+}$conformally onto $U$.

Lemma 1. Let $F$ be a function in $N^{+}$such that

(a) $F$ belongs to $H^{p}$ for some $p>0$,

(b) $F$ locally belongs to $H^{1 / 2}$ except a finite subset $A$ of $T$,

(c) $F$ is outer and $F\left(e^{i t}\right) \geq 0$ a.e. on $T$.

Then $F$ can be extended to a rational function.

Proof. By conditions on $F, F(z)$ can be extended beyond every boundary point in $T \backslash A$ to a holomorphic function on $C \cup\{\infty\} \backslash A$, which we also express by $F(z)$. Therefore if we can prove that each point $\alpha \in A$ is at most a pole of $F(z)$, it follows that $F$ is a rational function.

In the rest of the proof of this lemma, $M_{i}(i=1,2, \ldots, 5)$ stands for an appropriate positive constant. Let $\alpha$ be a point of $A$. To prove that $\alpha$ is a pole of $F$, we can assume without loss of generality that $\alpha=1$. If we put $G(z)=F(z)^{p}(1-z)^{2}$, then trivially $G(z) /(1-z)^{2}=F(z)^{p} \in H^{1}(U)$, and we have $G(\psi(z))=F(\psi(z))^{p}(1-\psi(z))^{2} \in H^{1}\left(C^{+}\right)$(cf. [4] p.130). From this we get

$$
|F(\psi(z))|^{p}|1-\psi(z)|^{2} \leq M_{1} \quad(\Im z \geq 1)
$$

and hence

$$
|F(\psi(z))| \leq \frac{M_{1}^{1 / p}}{|1-\psi(z)|^{2 / p}} \leq M_{2}|z|^{2 / p} \quad(\Im z \geq 1)
$$


By the conditions (b) and (c), it follows that $\bar{F}(\psi(z))=F(\psi(\bar{z}))$ on $C \backslash A$, and hence we get

$$
|F(\psi(z))| \leq M_{2}|z|^{2 / p} \quad(\Im z \leq-1)
$$

Next, since $F(\psi(z+a))^{p}(1-\psi(z+a))^{2} \in H^{1}\left(C^{+}\right)$for each $a>0$, we have by Fejer-Riesz inequality (cf. [2], p.46) modified to hold for a function in $H^{p}\left(C^{+}\right)$

$$
\begin{gathered}
\int_{0}^{\infty}|F(\psi(i t+a))|^{p}|1-\psi(i t+a)|^{2} \frac{1}{(1+t)^{2}} d t \\
\leq \frac{1}{2} \int_{-\infty}^{\infty}|F(\psi(x+a))|^{p}|1-\psi(x+a)|^{2} \frac{1}{1+x^{2}} d x \\
\leq \sup _{a>0} \int_{-\infty}^{\infty}|F(\psi(x+a))|^{p}|1-\psi(x+a)|^{2} d x \leq\|G \circ \psi\|_{1}<\infty
\end{gathered}
$$

Since

$$
\begin{gathered}
|1-\psi(i t+a)|=|1-(i t+a-i) /(i t+a+i)| \\
=|2 i /(i t+a+i)| \geq|1 /(i t+a+1)| \quad(t>0, a>0),
\end{gathered}
$$

we get

$$
\begin{aligned}
& \left.M_{3} \geq \int_{0}^{\infty}|F(\psi(i t+a))|^{p} \mid 1-\psi(i t+a)\right)\left.\right|^{2} \frac{1}{(t+1)^{2}} d t \\
& \geq \frac{1}{2} \int_{0}^{\infty}|F(\psi(i t+a))|^{p}\left|\frac{1}{i t+a+1}\right|^{4} d t \quad(a>0)
\end{aligned}
$$

Therefore if we choose $a>0$ large enough to assure $\Re \alpha<a \quad(\alpha \in A)$, we get that $F(\psi(z+a)) /\left.(z+a+1)^{4 / p}\right|_{C_{+}}$belongs to $H^{p}\left(C_{+}\right)$. From this we get

$$
|F(\psi(z))| \leq M_{4}|z|^{4 / p} \quad(\Re z \geq a)
$$

In the same way, we have

$$
|F(\psi(z))| \leq M_{5}|z|^{4 / p} \quad(\Re z \leq-b)
$$

for a large positive number $b$. From (3), (4), (7) and (8), we can conclude that $\infty$ is at most a pole of $F(\psi)$, that is $\alpha=1$ is at most a pole of $F$. This completes the proof of Lemma 1 . 


\section{REFERENCES}

1. deLeeuw, K. and Rudin, W., Extreme points and extremum problems in $H^{1}$, Pacific J. Math. 8.(1958), 467-485.

2. Duren, P. L., Theory of $H^{p}$ spaces, Academic Press Inc., New York and London 1970.

3. Garnett, J. B., Bounded analytic functions, Academic press. Inc. 1981.

4. Hoffman, K., Banach spaces of analytic functions, Prentice-Hall Inc., Englewood Cliffs, New Jersey 1962.

5. Inoue, J., An example of a non-exposed extreme function on the unit ball of $H^{1}$, preprint.

6. Koosis, P., introduction to $H^{p}$ spaces, Cambridge University Press, 1980

7. Nakazi, T., Exposed points and extremal problems in $H^{1}$, J. Funct. Anal. 53 (1983), 224-230.

8. Neuwirth, J. and Newman, D. J., Positive $H^{1 / 2}$ functions are constants, Proc. Amer. Math. Soc. 18 (1967) 958

Jyunji Inoue

Department of Mathematics

Faculty of Science

Hokkaido University

Sapporo 060

Japan
Takahiko Nakazi

Department of Mathematics

Faculty of Science

Hokkaido University

Sapporo 060

Japan 